\title{
El sonido de la seña inclusiva. Carteles fotográficos intervenidos y basados en la Lengua de Señas Mexicana
}

\author{
The sound of the inclusive sign. Photographic posters intervened and based on the Mexican Sign \\ Language
}

\author{
José C. Méndez-López ${ }^{a}$, María G. Buzo-Flores ${ }^{b}$, Ángel J. Petrilli-Rincón ${ }^{c}$
}

\begin{abstract}
:
This paper is about the results of the project Inclusive Culture, it was undertaken in two phases, and realized by teachers and students at the Fine Arts Faculty in the Universidad Veracruzana from Xalapa, Mexico, and with collaboration of the civil association Difussion, Inclusion and Education for Deaf (DIES in spanish). After a selection of nouns, verbs, animals, fruits, crafts and professions, we produced almost a hundred and twenty intervened photographic posters ${ }^{1}$ that allow visualizing by a kind of scanning flow the gestures and movements of each of those words as they occur in the Mexican Sign Language (LSM in spanish).

The production of posters, in general, were exhibited in two galleries in the city of Xalapa, Veracruz, Mexico, in order to move the population about this group and their communication using the Mexican Sign Language. The second phase, was directed to tourism sector and the words selected were useful verbs or nouns that helped them to recognize the needs of the deaf when they require tourist services. The posters were also exhibited at crowded public spaces like streets or alleys where population and tourists walk. The H. Ayuntamiento of Xalapa paid for the printing and gave the corresponding licenses.
\end{abstract}

\section{Keywords:}

Mexican Sign Language, deaf, inclusion, tourism, poster

\section{Resumen:}

El siguiente artículo muestra los resultados del proyecto Cultura Inclusiva, dividido en dos fases realizado por académicos y estudiantes de la Facultad de Artes Plásticas de la Universidad Veracruzana en colaboración con la asociación civil Difusión, Inclusión y Educación del Sordo (DIES). Después de seleccionar una serie de sustantivos, verbos, sentimientos, animales, frutas, oficios y profesiones, se realizaron alrededor de ciento veinte carteles fotográficos intervenidos ${ }^{2}$ que permiten visualizarlas por medio de un barrido que sigue los gestos y movimientos de cada una de las palabras en la Lengua de Señas Mexicana (LSM).

Los carteles producidos en el proyecto, en sus dos fases, se presentaron en dos galerías de la ciudad de Xalapa, Veracruz, México, para sensibilizar a la población en general sobre ese colectivo y su comunicación en LSM. La segunda, se orientó al sector turístico y las palabras elegidas fueron verbos o sustantivos que pudieran ayudar a conocer a dicho sector algunas de las necesidades de los sordos cuando requieren ciertos servicios turísticos. Los carteles resultantes se exhibieron en espacios públicos concurridos por los que transita, no sólo el peatón común, sino los turistas. El H. Ayuntamiento de Xalapa apoyó con la impresión y el permiso para exhibirlos en la calle y corredores peatonales.

\section{Palabras Clave:}

Lengua de Señas Mexicana, sordos, inclusión, turismo, cartel

\footnotetext{
${ }^{1}$ It's necessary to understand photographic intervention as a term used for any manipulated photography.

${ }^{2}$ Es menester mencionar que la intervención fotográfica es el término utilizado para toda la fotografía manipulada.
}

\footnotetext{
a José Cuauhtémoc Méndez López, Dr. en Ciencias y Artes para el Diseño, Responsable del Cuerpo Académico Arte y Transdisciplina UV-CA-385, Universidad Veracruzana, Facultad de Artes Plásticas, ORCID: 0000-0002-2509-3587, Email: jomendez@uv.mx

${ }^{\mathrm{b}}$ María Guadalupe Buzo Flores, Dra. en Historia del Arte, Integrante del Cuerpo Académico Arte y Transdisciplina UV-CA-385, Universidad Veracruzana, Facultad de Artes Plásticas, Email: gbuzo@uv.mx

c Ángel Javier Petrilli Rincón, Mtro. en Investigación en Psicología Aplicada a la Educación, Integrante del Cuerpo Académico Arte y Transdisciplina UV- 


\section{Objetivo}

Realizar carteles con palabras selectas de la Lengua de Señas Mexicana para sensibilizar al estudiante y al público sobre la lengua de sordos.

\section{Método}

Práctica con maestros y alumnos de DIES (Difusión, Inclusión y Educación del Sordo) para conocer la Lengua de Señas Mexicana. Se preparó un estudio fotográfico para realizar tomas de profesores y estudiantes universitarios, en forma organizada con profesores y estudiantes sordos para su supervisión de una seña ejecutada correctamente. Obteniendo como resultado del ejercicio el diseño de cartel fotográfico intervenido gráficamente con la representación visual de la palabra seleccionada. Todos los carteles se montaron en una exposición en una galería urbana y en espacio público.

\section{Introducción}

Vivimos en un mundo donde hay una variedad de personas en todos los aspectos: el físico, el ideológico, el religioso, el político, etcétera. Toda sociedad se ha caracterizado por la diversidad y ese aspecto la enriquece, pero eso no significa que siempre haya existido respeto por la variedad de colectivos humanos. Bien sabemos que todavía se lleva al cabo una lucha de varias minorías por conseguir un trato equitativo y respetuoso. Aún existen muchos atavismos en cuanto a diversidad se refiere y hay numerosos colectivos que son segregados en el ámbito social, económico, religioso, étnico, escolar y otros. La marginación se expresa de múltiples formas desde la falta de aceptación del "otro", el desprecio, la mofa o algún otro tipo de trato diferenciado con respecto a aquellos considerados "normales".

Un colectivo que ha padecido la incomprensión, e inclusive la segregación, es el de las personas con discapacidad auditiva, ya sea parcial o total. Aquí se presenta un panorama que concierne a dicha comunidad, es decir, cuántas personas la padecen en el mundo y en México y cómo la Lengua de Señas Mexicana (LSM), que es la más difundida en el país, sirve para que se comuniquen; sin embargo, el resto de la población, desafortunadamente, no está familiarizada con dicha lengua ni tampoco sensibilizada. Por esa razón, se decidió colaborar con la asociación civil Difusión, Inclusión y Educación del Sordo (DIES) que opera en la ciudad de Xalapa. Esta organización busca mejorar las condiciones de vida e inclusión de las personas sordas a través de la capacitación y educación para conseguir un reconocimiento y respeto de ese colectivo y de la LSM.

Se trabajó con maestros de DIES, quienes acudieron a la Facultad de Artes Plásticas de la Universidad Veracruzana, para enseñar a algunos docentes y varios grupos de alumnos el sentido de la LSM. Lo primero que se hizo fue una sensibilización para comprender la problemática cotidiana a la que se enfrentan los sordos. Se hizo una selección de palabras que los estudiantes trasladaron a carteles fotográficos intervenidos. Con el auxilio de los maestros de LSM, consiguieron hacer un "barrido visual" de los gestos y movimientos de esas palabras para presentarlos en los carteles. Tras concluir la producción, el resultado se presentó en un espacio de exposiciones para sensibilizar a la población en general sobre este panorama.

Después de esa primera experiencia y encuentro con personal de DIES y con personas sordas apoyadas por ellos, la fase siguiente del proyecto consistió en involucrarse en la cultura de la inclusión turística. Puesto que ya se cuenta con una legislación que apoya el turismo inclusivo, se trabajó sobre dicho tema y se consiguió el apoyo del H. Ayuntamiento de Xalapa y de la Secretaría de Turismo y Cultura de Veracruz que financiaron la impresión de una nueva serie de carteles con palabras alusivas a los servicios y al turismo inclusivo. Se realizaron alrededor de ciento veinte carteles con palabras útiles para una visita turística de ese colectivo.

Se presenta a continuación las reflexiones teóricas, legales, de educación e inclusión relacionadas con la comunidad sorda, la LSM y los resultados conseguidos con este proyecto inclusivo.

\section{Desarrollo}

\section{Cultura inclusiva}

Entendemos la cultura como un proceso ininterrumpido de producción de comunicación y de significación, en transformación y actualización, que es compartido en la práctica individual y colectiva en contextos específicos histórica y socialmente estructurados. La cultura, comprendida como acción social por agentes sociales concretos dota de sentido las interpretaciones y los significados de cada comunidad o subcultura. La cultura se interioriza, se organiza en el sujeto y se refleja en las 
distintas representaciones sociales y contribuye a forjar la identidad (Giménez, 2005).

Uno de los mayores retos sociales para conseguir una cultura de la diversidad y la inclusión, implica que sea la sociedad la que cambie sus comportamientos y actitudes con respecto a los colectivos marginados para que dejen de verse sometidos a la tiranía de la normalidad, como la denomina (López, 2011). Una cultura inclusiva pretende llegar a otros agentes sociales para quienes ciertos significados no son conocidos.

Hablar de cultura inclusiva, no sólo es referir a la cultura de capacidades diferentes, sino que es crear una conciencia en el ser humano y reforzar sus valores, los cuales hoy en día están abandonados en un rincón donde todos los ven y nadie los practica.

En lo que llevamos de este siglo son cada vez más las iniciativas e investigaciones que abordan el tema de la diversidad, la marginación de minorías y la necesidad de fomentar una cultura de inclusión. Una cultura inclusiva parte de la idea de brindar oportunidades equivalentes para todas las personas en lo educativo, lo social, lo laboral, los servicios, lo cultural.

En el plano social resulta todavía más arduo conseguir un trato equitativo y respetuoso para las personas con hándicaps. Es muy común que se las segregue y se las considere como "discapacitadas" o, en el mejor de los casos, como suele decirse hoy día "con capacidades diferentes". Esta manera de verlo esconde prejuicios, además de una diferenciación con respecto a las personas consideradas "normales". La exclusión resulta evidente en nuestro entorno cotidiano en las ciudades y en los poblados que no cuentan siquiera con la infraestructura mínima para personas con hándicaps. Frente a este panorama consideramos que es una obligación de quienes nos dedicamos a la educación superior, sensibilizar a los alumnos y crear conciencia de esta problemática, no sólo educativa, sino social.

\section{Educación inclusiva}

La educación y la cultura inclusiva fomenta un proceso de humanización, tan necesario en nuestros días, supone comprensión y cortesía, participación y convivencia. Es un camino para aprender a vivir con las diferencias de las personas y aportar equidad y sentido ético para combatir la segregación y la discriminación. Implica empatía con nuestros semejantes. Quedan muchas barreras por superar. $\mathrm{Y}$ una forma de propiciar un modelo educativo más ad hoc con las metas inherentes para una educación y cultura inclusiva en este siglo, consiste en plantear al estudiante problemas reales, cercanos a su entorno, es decir, aprender a analizar situaciones que propicien propuestas creativas, cognitivas y emocionales que lo concientice y lo sensibilice sobre problemáticas y/o situaciones reales. Esta modalidad de aprendizaje aporta no sólo conocimiento sobre su entorno sino que lo obliga a aportar soluciones como un profesional. Al participar activamente en la sociedad el alumno será más solidario y comprensivo con los distintos grupos y frente a variadas circunstancias. De este modo, el discente será capaz de asimilar mejor su entorno social, de analizar las causas de las distintas formas de discriminación y además de tomar conciencia, incorporarse de manera activa en la propuesta de soluciones que ayuden a construir una educación y una cultura inclusiva.

Hablar de cultura inclusiva en la educación es efervescer los valores y modificar los prejuicios y estereotipos o modelos mentales con los que docentes y padres de familia han crecido, reproduciendo constantemente algunas prácticas muy poco acertadas para la formación integral de los estudiantes e hijos y empoderar así nuestros pensamientos, hechos y palabras para que sean la clave para construir una atmósfera escolar favorable en donde los académicos sean el reflejo de la práctica y rescate de valores tales como; el respeto, la colaboración, la justicia y la identidad. Se define la cultura inclusiva como aquella centrada

... en crear una comunidad segura, acogedora, colaboradora y estimulante en la que cada uno es valorado, como el fundamento primordial para que todo el alumnado tenga los mayores niveles de logro. Pretende desarrollar valores inclusivos, compartidos por todo el profesorado, el estudiante, autoridades o miembros de los cuerpos colegiados o los académicos del consejo escolar y padres de familia, que se transmitan a todos los nuevos miembros del centro educativo (Booth \& Ainscow, 2002, p.16).

La Universidad, como Institución educativa, debe cumplir con una función académica y socializadora (enfatizando su visión principal en tener que brindar resultados centrados en una educación de calidad). Retomamos básicamente lo esencial del informe de monitoreo de la (UNESCO Educación para Todos, 2005), en el que se establecen tres elementos principales para definir la educación de calidad: respeto de los derechos de las personas; equidad en el acceso, procesos y resultados y pertinencia de la educación. Responder con esos criterios involucra la calidad, y cumplir con la función socializadora implicará favorecer el manejo de conflictos de manera pacífica y permitir la 
toma de decisiones y la autorregulación de las emociones, las cuales se ven reflejadas en una convivencia armónica. En ese orden de ideas, destacamos que "la educación inclusiva, conocida también como educación integradora, se basa, ante todo, en el derecho de cada individuo a la educación, inscrito en el Artículo 26 de la Declaración Universal de Derechos Humanos de 1948 (UNESCO, s/f). Sin embargo, queda mucho por hacer para conseguir una transformación de los sistemas educativos que haga frente a la diversidad de los educandos y que abarque los cuatro pilares de la educación propuestos para este siglo: aprender a conocer, aprender a ser, aprender a hacer y aprender a convivir.

La Asociación Nacional de Universidades e Instituciones de Educación Superior (ANUIES) en México, publicó un Manual para la integración de personas con discapacidad, en el que presenta las acciones básicas para transitar hacia la incorporación de los grupos humanos menos favorecidos por contar con alguna discapacidad. Sus propuestas parten de un enfoque multicultural, inclusivo y de comunidad en el que todos los involucrados se sientan aceptados, se apoyen entre sí de acuerdo con sus capacidades en un ambiente de respeto a la dignidad humana y a las diferencias.

En el 2010, se aprobó el Plan Maestro para la Sustentabilidad de la Universidad Veracruzana que, contribuye a generar conocimiento y formar universitarios desde una perspectiva social con respeto a las diferencias. Siguiendo los lineamientos, la visión y misión del mismo es que se desarrolló este proyecto, en dos fases, con alumnos de la Facultad de Artes Plásticas, colaborando con la asociación civil Difusión, Inclusión y Educación del Sordo (DIES), así como con la Secretaría de Turismo del Estado de Veracruz para sensibilizar y concienciar en torno a la problemática de segregación que la comunidad Sorda padece, en aras de propiciar el conocimiento de la Lengua de Señas Mexicana (LSM).

\section{Población con discapacidad}

De acuerdo con el Informe Mundial sobre la Discapacidad, casi 200 millones de personas experimentan dificultades en su funcionamiento, y en los años venideros será motivo de preocupación aún mayor, debido al envejecimiento de la población, así como al aumento de enfermedades crónicas y de trastornos de la salud mental (OMS, Banco Mundial, 2011). Muchas de estas personas experimentan problemas de inserción social, peores resultados sanitarios y académicos, así como exclusión en el mercado laboral y de servicios por lo que su accesibilidad a una vida plena se ve disminuida. De ahí que, la OMS recomiende invertir en programas y servicios específicos para las personas con discapacidad, adoptar medidas y planes de acción nacionales, mejorar la capacidad de los recursos humanos a los profesionales de prestación y gestión de servicios y fomentar la sensibilización pública y la comprensión de la discapacidad, entre otras.

En cuanto a los problemas de discapacidad auditiva, la OMS (2018) menciona que, en 2018, 466 millones de personas en el mundo padecen pérdida de audición discapacitante que se entiende como superior a $40 \mathrm{~dB}$ en adultos y superior a $30 \mathrm{~dB}$ en niños. Las personas con discapacidad auditiva grave "pueden aprender a comunicarse mediante la lectura de los labios, los textos escritos o impresos y el lenguaje de signos".

En el caso de México, los datos del INEGI, en 2010, reportan que, la población con algún tipo de discapacidad, ascendía a 5 millones 739 mil 270, lo que representa el $5.1 \%$ del total de población, de los cuales los que presentan discapacidad auditiva son el $12.1 \%$, es decir, 474 mil 319 personas. Para conseguir una sociedad inclusiva hace falta trabajar arduamente; $y$, en ese sentido, el Consejo Nacional para Prevenir la Discriminación (CONAPRED), ha publicado una guía de reflexión, análisis y acción que define criterios para comprender y examinar las problemáticas relacionadas con la discriminación y orienta a las instituciones para una accesibilidad integral, inclusión y diversidad. Esta tarea es fundamental para contar con una sociedad más justa e inclusiva.

\section{Algunas lenguas de señas en el mundo}

Las personas sordas han sido un sector de población que, a lo largo de la historia, ha sido imperceptible, y hasta incómodo para la población hablante. Han sido discriminados en muchos aspectos de la vida; y cuando de educación hacia ellos se trata, hay estudios que demuestran que existe una relación entre la educación de los sordos y las señas utilizadas por ellos y sus maestros. Una de las iniciativas más antiguas de las que se tiene conocimiento destaca el papel del fraile benedictino Pedro Ponce de León, en España, quien intentó con éxito educar al sordo en el siglo XVI; mientras que, en el siglo XVII se publicó en Sevilla un texto para educar al sordo considerado en primero en su género en el mundo (Cruz, 2008).

En 1760, el abad Charles Michel de L’Epée, comenzó a utilizar un lenguaje de señas para comunicarse con sordos y fue el fundador del Instituto Nacional de Sordos que dio lugar a la Lengua de Señas Francesa (LSF). En 1880, se organizó en Milán un 
"Congreso internacional para la mejora del destino de los sordomudos", en el que se votó la resolución de la superioridad del método oral sobre la lengua de señas. Desde entonces se prohibió el uso de la lengua de señas en la educación de los sordos y sus consecuencias han sido perceptibles en los distintos lugares del mundo, dado que, se dejaron de lado y/o cayeron en desuso las lenguas de señas. Tuvo que pasar mucho tiempo para que la comunidad Sorda de cada país, presionara socialmente para que, finalmente, se reconociera que las lenguas de señas son lenguas naturales que cuentan con una gramática y sintaxis como cualquier lengua humana.

La American Sign Language (ASL), es la lengua de señas usada en los Estados Unidos por los sordos o personas con graves problemas de audición, se estima que tiene más de doscientos años en uso y es una mezcla entre lenguas de signos locales y algunos elementos de la Lengua de Señas Francesa (LSF), aunque no se sabe exactamente cuándo inició $(\mathrm{NIH}$, 2017). Otras lenguas de señas con influencia de la LSF son Lengua de Señas Holandesa, la Lengua de Señas Brasileña, la Lengua de Señas Australiana o la Lengua de Señas Mexicana, entre otras.

Aunque la investigación lingüística de las lenguas de señas es reciente, pues solo se remonta a mediados del siglo pasado (Cruz, 2009), es notorio que hoy día, se cuenta con más investigaciones sobre ellas. El resurgimiento de las lenguas de señas en Europa y algunos países del continente americano despunta con fuerza hacia los años 90 del siglo pasado, dejando a un lado el anterior oralismo. Actualmente hay trabajos sobre la estructura, la semántica y la sintaxis de alguna lengua de señas particular, así como estudios comparativos,$^{\dagger}$ sobre la educación en lengua de señas ${ }^{\ddagger}$, en síntesis, las lenguas de señas cuentan con un reconocimiento que ha beneficiado la inclusión de las comunidades de sordos, así como su educación. Y si bien, se pueden reconocer los grandes avances, hay mucho que todavía queda por hacer para erradicar de la

\footnotetext{
* La asistencia de miembros al congreso varía según la fuente, se habla de 255 o 167 votantes. A detalle, estuvieron presentes ciento cincuenta y seis italianos (todos favorables al método oral), sesenta y seis franceses, doce ingleses, ocho alemanes, seis estadounidenses, un belga, un suizo, un canadiense, un ruso y un sueco; de los cuales solo tres eran sordos (Encrevé, 2008) (traducción propia).

† Véase el estudio comparativo de la ASL con las lenguas de señas japonesa y china. Fischer, S. (2003). "The Cross-Linguistic Study of Sign Languages", TCT Education for Disabilities, 2, https://www.tsukubatech.ac.jp/repo/dspace/bitstream/10460/106/1/ETec02 0 01.pdf, fecha de consulta: 18 de abril de 2018 .

\$ Mertzani, M. (2015). "How far have we gone with Applied Sign Linguisticsin deaf education?", Pro-Posições, 26 no.3, http://www.scielo.br/scielo.php?, fecha de consulta: 18 de abril de 2018 .
}

mente de la gente común el desprecio o discriminación a dicha comunidad.

\section{Lengua de Señas Mexicana (LSM)}

Toda lengua de señas se vale de la especialidad corporal de quien emite las señas que, activa movimientos visogestuales, por medio de las manos y del cuerpo y que sigue una serie de normas gramaticales particulares relacionadas con la lengua de señas específica aprendida. Las lenguas de señas no son universales, sino que se han desarrollado de formas diversas obedeciendo en cada país a situaciones histórico sociales concretas.

Al conocer cada vez más las lenguas de señas, la función del espacio se ha revalorado como un elemento fundamental del sistema de las lenguas de señas. Pues no sólo basta el reconocimiento del papel de los articuladores, como las manos, el cuerpo y la cabeza, sino que además la dimensión del espacio que el señante tiene enfrente de su cuerpo cumple un papel en la forma de los signos y de las construcciones gramaticales. (Cruz, 2008, 15)

Los Sordos hablan con su propia lengua, lo que les da identidad y de ese modo pueden comunicarse y trasmitir su cultura. Las lenguas de señas son lenguas naturales y poseen una gramática y una sintaxis como cualquier otra lengua. Las lenguas de señas, en el continente americano, que tienen su origen en un método de signos metódicos para sordos que surgió en París hacia 1760 son, entre otras, la LSM y su equivalente en Brasil (LIBRAS).

La educación del sordo en México guarda relación con la Ley de instrucción decretada por Benito Juárez, en 1861, para establecer una escuela para sordomudos. La fundación de la Escuela Nacional de Sordomudos se hizo efectiva en 1867 y su fundador y director fue Eduardo Huet, un francés que había quedado sordo y conocía la lengua de señas. Él mismo había sido el fundador de la primera escuela de sordomudos en América Latina, en el año 1852 en la ciudad de Río de Janeiro. La escuela mexicana contó con el apoyo de Maximiliano, y cuando Juárez regresó al poder tras el triunfo de la República continuó apoyando esta sede educativa (Cruz, 2008).

Cabe señalar que, en $1880, \S$ se enfrentaron dos propuestas distintas de la enseñanza para sordos: la

\footnotetext{
$\S$ Año en que se realizó un congreso en Milán que dejó de manifiesto la preferencia por la oralidad para la inserción del sordo a la sociedad (Cruz, 2009).
} 
alemana hacía énfasis en la adquisición por parte del sordo, de la oralidad y la lectura de los labios; mientras que la tendencia francesa que privilegiaba la lengua de señas cayó en desuso. Este cambio de enfoque se reflejó también en México donde la educación del sordo tendió a la oralidad y la enseñanza de la lectura y la escritura. Tuvo que pasar mucho tiempo para que se reconociera que la LSM es una lengua natural como cualquier otra y fue gracias a la presión social de la comunidad sorda que finalmente el poder ejecutivo emitió una ley que reconoce los derechos del sordo y su lengua.

\section{Legislación con fines inclusivos en México}

El reconocimiento de la LSM se dio en México, en 2005, en el marco de la Ley General de personas con discapacidad publicado en el Diario Oficial de la Federación, cuya última reforma se publicó en diciembre de 2015. A continuación destacamos algunos de los incisos del artículo 2, que explican qué se entiende por

V. Comunicación. Se entenderá el lenguaje escrito, oral y la lengua de señas mexicana, la visualización de textos, el sistema Braille, la comunicación táctil, los macrotipos, los dispositivos multimedia escritos o auditivos de fácil acceso, el lenguaje sencillo, los medios de voz digitalizada y otros modos, medios, sistemas $y$ formatos aumentativos o alternativos de comunicación, incluida la tecnología de la información y las comunicaciones de fácil acceso;

VI. Comunidad de sordos. Todo aquel grupo social cuyos miembros tienen alguna deficiencia del sentido auditivo que les limita sostener una comunicación y socialización regular y fluida en lengua oral....

XVII. Lengua de Señas Mexicana. Lengua de una comunidad de sordos, que consiste en una serie de signos gestuales articulados con las manos y acompañados de expresiones faciales, mirada intencional y movimiento corporal, dotados de función lingüística, corma parte del patrimonio lingüístico de dicha comunidad y es tan rica y compleja en gramática y vocabulario como cualquier lengua oral $(2011,2,3)$.

En la misma ley se determinó fundar el Consejo Nacional para el Desarrollo y la Inclusión de las Personas con Discapacidad (CONADIS) que promueve el diseño y la instrumentación de programas para la inclusión en los ámbitos de derecho a la salud, la educación, el trabajo, la accesibilidad, el transporte, el deporte, el turismo y la cultura.

Ese mismo año Francia decretó la ley 2005-102, por la igualdad de los derechos y oportunidades, la participación y la ciudadanía de las personas con discapacidad, poniendo fin al conflicto previo que privilegió la inserción del sordo a través de la oralidad. Dicha legislación se orienta hacia la no discriminación, la inserción profesional, el derecho a la educación, a la autonomía, la participación en la vida social y política de las personas con discapacidad.

En México, el año 2009, se decretó la Ley General de Turismo que en el artículo 2, inciso VI, determina facilitar que las personas con discapacidad puedan disfrutar de las instalaciones destinadas a la actividad turística y contar de ese modo con un turismo accesible según consta en el Capítulo IV, artículos 18 y 19, que indican que los prestadores de servicios deberán proveer lo necesario para aquellos con discapacidad (DOF, 2015).

La Guía de acción contra la discriminación se publicó en 2011. Consta de una serie de apartados que orienta a los organismos, instituciones, empresas y escuelas para comprometerse en planes de acción que redunden en mecanismos y propuestas que involucren a todas las personas que forman parte de nuestra sociedad sin distingo de género, apariencia física, extracto social, origen, preferencia sexual, discapacidad - cualquier otra variable que pudiera generar segregación o sectarismo. Entre sus objetivos está fomentar el desarrollo y las acciones para la igualdad y la no discriminación, además de sensibilizar a la población sobre la inclusión, accesibilidad y diversidad e igualdad (CONAPRED).

El estado de Veracruz, por su parte, emitió en la Gaceta Oficial del 11 de marzo de 2010, la Ley para la integración de las personas con discapacidad del Estado de Veracruz de Ignacio de la Llave. En ella, se enlistan los criterios que definen jurídicamente qué entender por discapacitados, comunicación, comunidad de sordos y discapacidad sensorial, entre otros; y también propone que se brinde igualdad de oportunidades en todos los ámbitos a dichas personas. Además, reconoce como un lenguaje la LSM, lo que finalmente implica un avance y una propuesta inclusiva para la comunidad sorda.

\section{Por un turismo inclusivo}

Las personas con discapacidad se enfrentan a obstáculos de distinta índole, desde políticas públicas y normas insuficientes o que simplemente se quedan en el 
papel, incomprensión y prejuicios, prestación de servicios no adaptada a las diferencias de estos colectivos y falta de capacitación de profesionales y prestadores de servicios.

La Organización Mundial del Turismo (OMT) se encuentra comprometida con la promoción y divulgación de un turismo accesible, es decir, que los productos y servicios turísticos se encuentren al alcance de todos los usuarios. Los países firmantes de la Convención de las Naciones Unidas sobre los derechos de las personas con discapacidad deben seguir las recomendaciones para que la experiencia turística de cualquier persona resulte satisfactoria. Para ello es imprescindible contar con accesibilidad a los destinos, transporte adecuado, medidas de seguridad, información en formatos accesibles, personal capacitado (2016).

La importancia del turismo en México es una fuente de derrama económica para pequeños, medianos o grandes empresarios y prestadores de servicios. Se calcula que representa el 8.5\% del PIB (OCDE, 2017, p. $5)$. Este documento detecta varias problemáticas y los desafíos para un turismo sustentable y accesible. En particular, relacionado con la comunidad sorda, menciona la necesidad de promover el crecimiento de un turismo incluyente.

Tanto la legislación nacional como las propuestas e iniciativas de acuerdos internacionales coinciden en propiciar, promover y sugerir soluciones sobre las distintas problemáticas para ofrecer servicios turísticos accesibles para todos; sin embargo, en nuestro país resulta evidente que hay un rezago lamentable y que la accesibilidad turística no siempre es factible. Pudiera pensarse que el turismo para la comunidad sorda resultara más accesible que para invidentes o personas con problemas de movilidad, sin embargo, hay que considerar que no estamos familiarizados con la lengua de señas, lo que repercute en situaciones de estrés para un sordo cuando intenta comunicarse con otros.

A partir de todo ese proceso de reflexión planeación y diseño surge el proyecto de Cultura Inclusiva que se desarrolló en dos periodos distintos, con la participación de estudiantes de las Licenciaturas Artes Visuales, Diseño de la Comunicación Visual y Fotografía de la facultad de Artes Plásticas de la Universidad Veracruzana en forma colaborativa y con la asesoría de DIES (Difusión, Inclusión y Educación del Sordo). En la primera parte, sólo se diseñaron una serie de carteles basados en las tomas fotográficas -barridorepresentando una serie de palabras (profesiones, oficios, sentimientos y frutas) intervenidas gráficamente con una ilustración digital que reforzaba la seña representada.

Los resultados se mostraron a través de exposiciones en la galería del Jardín de las Esculturas del Instituto Veracruzano de Cultura, en Xalapa, así como en otros espacios del estado de Veracruz, México. La segunda fase del proyecto surge a petición del $\mathrm{H}$. Ayuntamiento de Xalapa a través de la Secretaría de Turismo del Estado de Veracruz los diseños se trabajaron con la misma técnica, palabras relacionadas con el turismo cultural. Ejemplos: Habitación, cuenta, propina, wifi, leche, jugo, desayuno, alberca, bata de baño, doctor, check in, check out. Cerrando la actividad exhibiendo todos los carteles en la galería Cultural del Centro Recreativo Xalapeño.

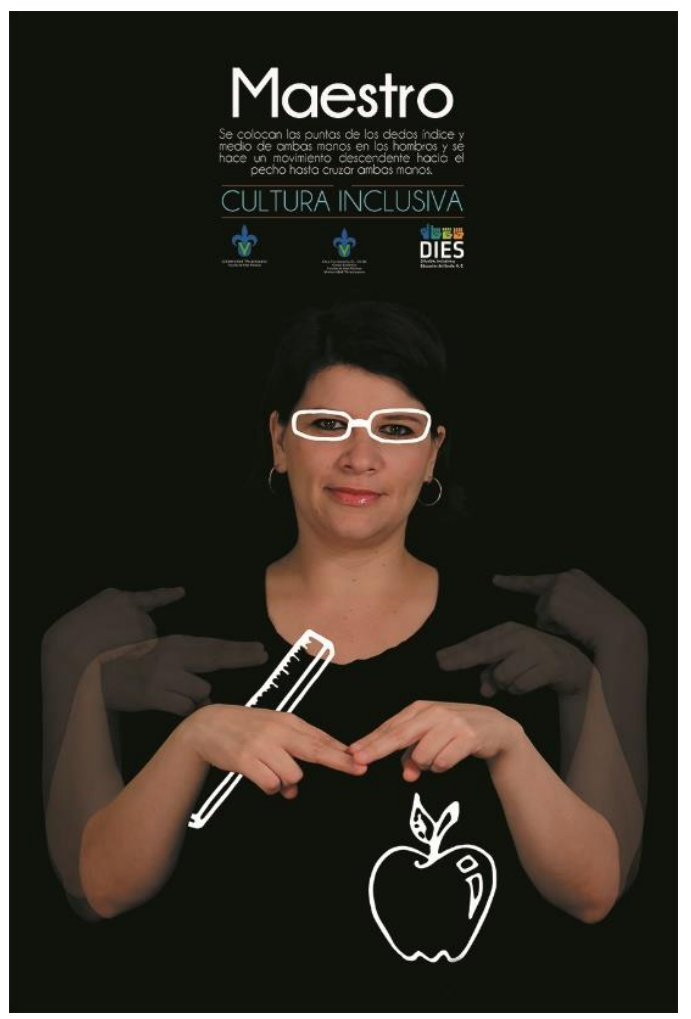

Figura 1. Maestro Fuente: Méndez 2018. 


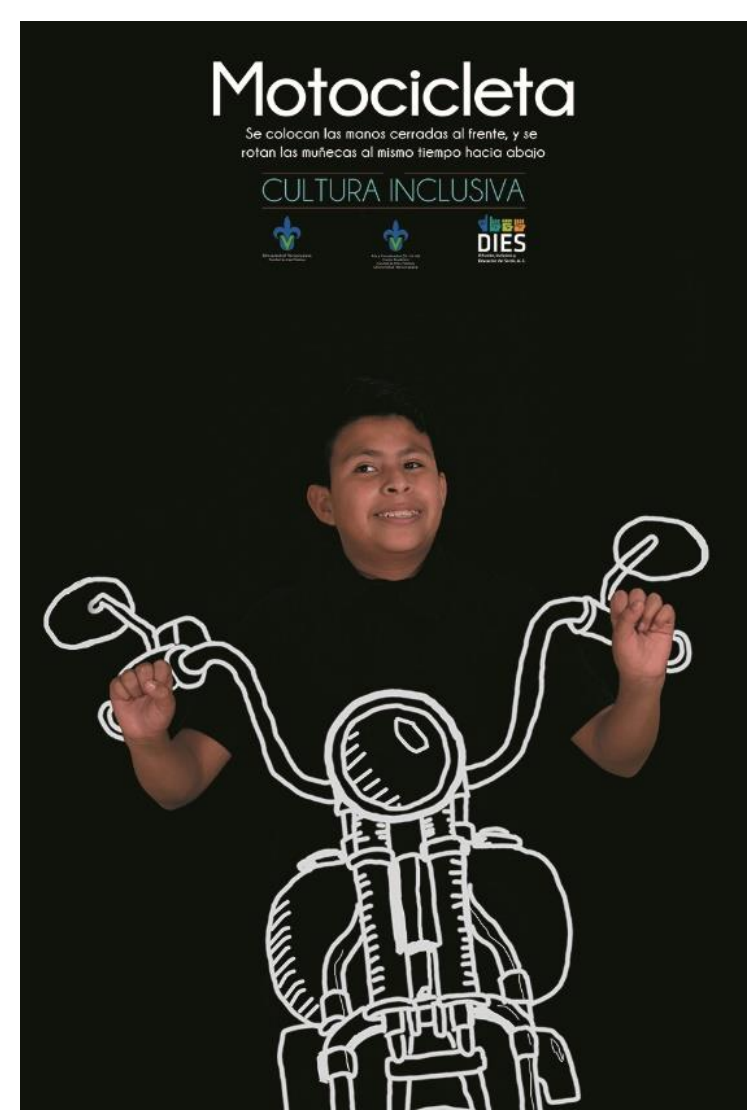

Figura 2. Moto Fuente: Méndez 2018.

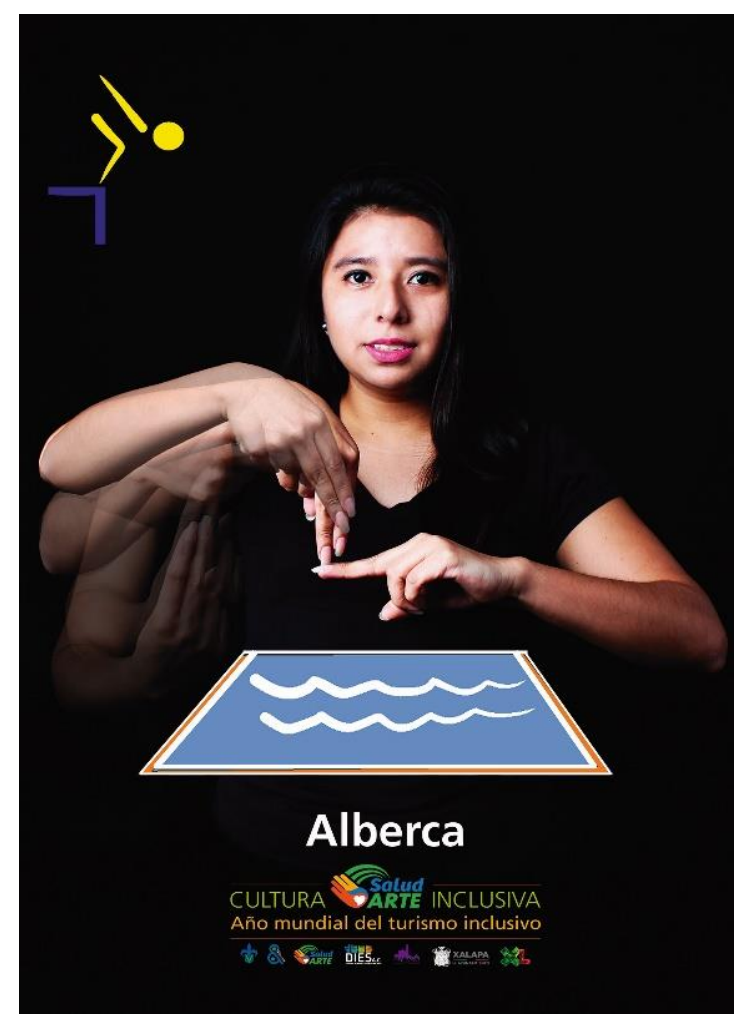

Figura 3. Alberca. Fuente: Méndez 2018.
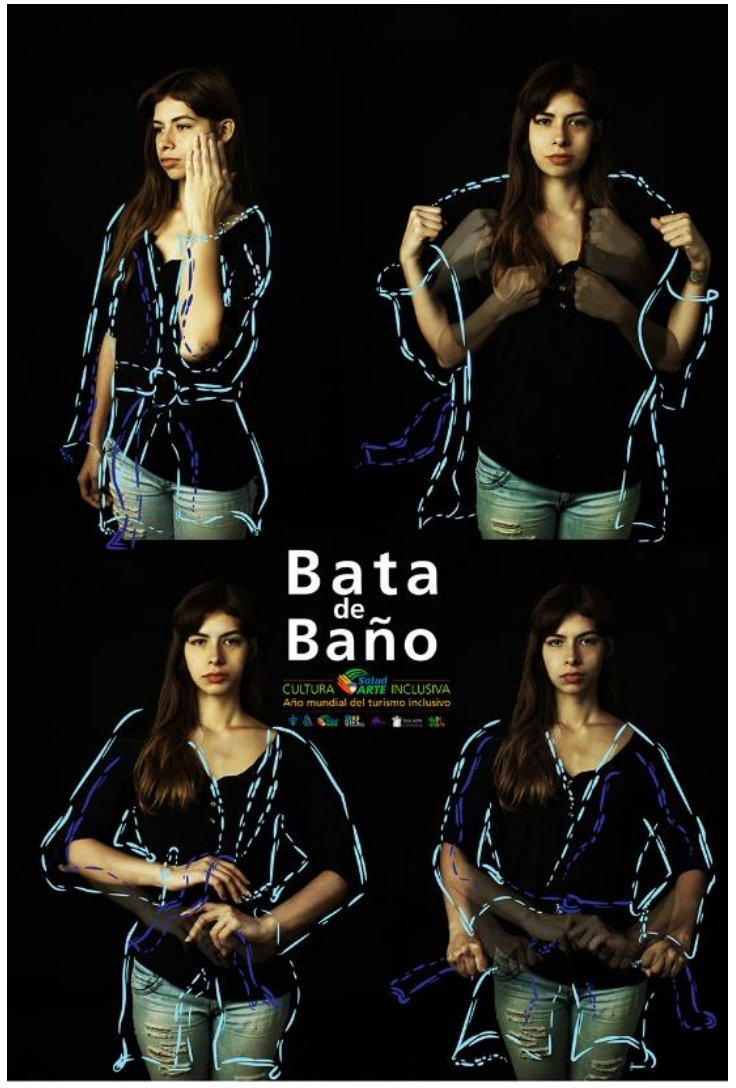

Figura 4. Bata de baño. Fuente: Méndez 2018.

\section{Carteles fotográficos intervenidos}

Bajo esta serie de premisas, el Cuerpo Académico Arte y Transdisciplina de la Facultad de Artes Plásticas de la Universidad Veracruzana diseñó el proyecto Conociéndonos: cultura inclusiva, junto con la Asociación Civil DIES (Difusión, Inclusión y Educación del Sordo) que tuvo como objetivo principal impulsar el desarrollo de las políticas, culturas y prácticas inclusivas en los estudiantes, profesores y público en general a través de formas de colaboración y modelos de gestión entre la comunidad educativa como un mecanismo de interacción y orientación que ha permitido el diseño de estrategias de atención a fin de responder a la diversidad, además de eliminar las barreras del aprendizaje y la acción entre los participantes.

En este proyecto se trabajó en forma conjunta y con la ayuda de profesores y niños sordos quienes asisten a dar y tomar clases a la asociación DIES, para desarrollar en su primera fase, una serie de carteles considerando verbos, sentimientos, oficios y frutas como temas principales y en su segunda fase el tema del turismo cultural. En la realización se involucraron los estudiantes de dos Experiencias Educativas. Con la finalidad de planear de forma creativa formas de 
atención inclusiva que como resultado dio el diseño de carteles con intervenciones gráficas-ilustraciones que auxiliaran en forma semántica descriptiva al público general y fundamentado en la Lengua de Señas Mexicana (LSM), que es el medio de comunicación usado en la comunidad Sorda en las regiones urbanas y rurales de México.

Durante el proceso y asumiendo el compromiso universitario de establecer una conexión entre los conocimientos y los aprendizajes del currículo con la sociedad, se propuso a los alumnos un ejercicio de sensibilización con respecto a las personas sordas. Consistió en conocer algunas de las problemáticas a las que se enfrentan, así como un acercamiento al conocimiento de la LSM, para lo cual se contó con el apoyo de profesores del DIES. Los estudiantes diseñaron una serie de carteles que dan a conocer algunas palabras. La solución visual es sencilla y clara y está complementada con un texto explicativo de cada una de las palabras, además de contar de forma visual con un barrido de los movimientos para cada palabra en la LSM. Se impulsó el desarrollo de prácticas inclusivas entre los estudiantes, profesores y público en general en colaboración con niños con deficiencia auditiva que acuden a esa asociación civil para aprender un lenguaje de señas por medio del cual establecer una comunicación con las demás personas.

Se seleccionaron palabras de uso cotidiano que comprenden verbos, objetos, oficios, frutas $y$ sentimientos para crear un número equivalente de carteles que enfatizan el lenguaje usado por esa comunidad específica. Los carteles se mostraron en una exposición en una galería de la ciudad de Xalapa en diciembre de 2016. La exhibición, sorprendió por igual a quienes la visitaron. La calidad del trabajo del conjunto de quienes colaboraron en él resulta evidente en su diseño, su sencillez, su facilidad de comprensión, su equilibrio y armonía. Cumple, por tanto, con los requisitos inherentes a un buen diseño de cartel: eficacia, fuerza creativa, vigor en su solución, simplicidad de formas, equilibrio en su composición y manejo cromático con lo cual es posible promover la aceptación de ese sector de población marginado, de sensibilizar y concientizar para propiciar la aceptación social y potenciar, por ende, una cultura inclusiva y flexible que llegue a redundar en la aceptación de todas las personas con cada una de sus diferencias. Figura 5. Wifi. Figura 6. Doctor.

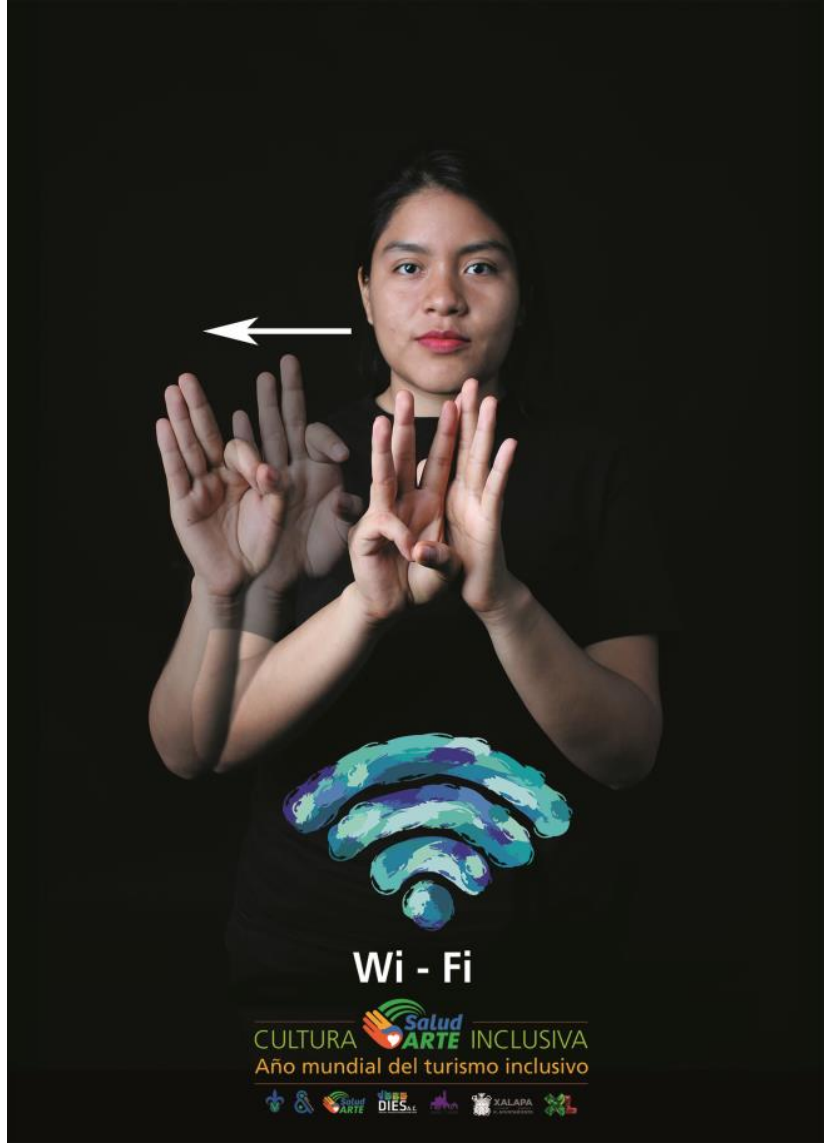

Figura 5. Wi Fi. Fuente: Méndez 2018.

Las fotografías base de los carteles, fueron compuestas y diseñadas por los estudiantes del 1er semestre de la Licenciatura en Diseño de la Comunicación Visual; mientras que, las intervenciones gráficas-ilustraciones de verbos, sentimientos, frutas, oficios y profesiones y en particular una serie sobre el tema del turismo cultural, las diseñaron los estudiantes de semestres avanzados de la Licenciaturas Diseño de la comunicación Visual, Artes Visuales y Fotografía.

La intervención se puede acercar a dos mundos: uno es el mundo individual, la creación de cada estudiante, su estética, tendencia, su propio lenguaje comunicativo, el otro mundo aparece al tomar distancia sin despegar la mirada y el cuerpo; detalles y signos enlazan el contenido semántico, es la fascinación que cada individuo siente por lo que fotógrafo son esos cuerpos, objetos, paisajes, reflejando el deseo, la indiferencia, el odio, la incertidumbre, la libertad, la incesante e inmanente búsqueda de hacer conciencia a quienes observan el resultado. 


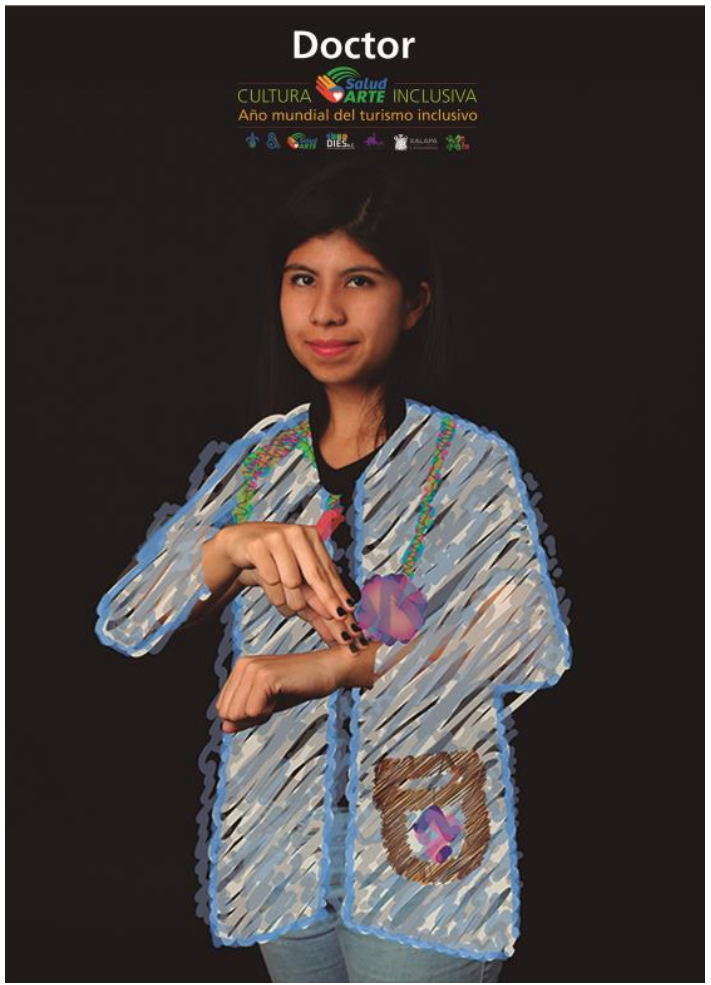

Figura 6. Doctor. Fuente: Méndez 2018.

Los estudiantes tuvieron la oportunidad de manifestar sus conocimientos a través de la práctica del desarrollo cultural inclusivo comunitario, vinculándose con el arte comunitario o de la mediación artística (MA), término propuesto en el año 2006 por Jean Pierre Klein, quien afirma que el "Arteterapia" puede llamarse Psicoterapia de Mediación Artística, siendo el arte un mundo entre otros o una técnica como los medicamentos (Klein, 2006, p.7). Y de la función estética y pedagógica a la función social y las terapéuticas, posteriormente acuñado por Ascención Moreno, proviene de las confluencias de las ciencias de la educación social, a la psicología, la arteterapia, la educación artística, la filosofía y la animación sociocultural. (López, 2013).

La mediación artística: un modelo de educación artística para la intervención social a través del arte. Moreno, llama a este modelo mediación artística, porque pone el acento en la idea de que el arte es una herramienta, es decir; el medio que posibilita la experiencia de reinserción y transformación social. EI arte media entre la identidad de cada persona y el entorno, entre sus valores e ideas, que no son abordadas directamente, sino a través de producciones culturales u objetos artísticos como lo es en este caso la producción de carteles intervenidos gráficamente.

\section{Conclusión}

El impacto que tuvieron estos dos proyectos fue el de sensibilizar y concientizar primero a los estudiantes y docentes involucrados sobre la comunidad de sordos y conocer, aunque sea sucintamente, la importancia de la comunicación por medio de su lengua: la Lengua de Señas Mexicana. Además, se contó con un público numeroso que acudió a visitar la exposición de carteles fotográficos intervenidos que se realizaron en el primer proyecto. Hubo buena afluencia a la galería y la exhibición fue muy bien valorada y recibida por los visitantes.

En el terreno del aprendizaje, las actividades colaborativas ayudan a los estudiantes a conocerse y a confiar entre ellos y sobre todo a empezar a incluir y desarrollar otras habilidades sociales importantes, como son aceptar y apoyar a los demás en la resolución de problemas de una forma constructiva. De manera entonces importante, a los académicos como facilitadores de los procesos, nos incluye modelar habilidades interpersonales positivas y además hacer que los estudiantes practiquen dichas habilidades para que estas sean significativas.

Un gran logro fue contar, para el segundo proyecto inclusivo, con la aprobación y apoyo del $\mathrm{H}$. Ayuntamiento del Municipio de Xalapa que se involucró y asumió el costo de la impresión de los carteles con temas referentes a los servicios para propiciar un turismo inclusivo. Además de la atinada difusión que se dio al haber otorgado el permiso para presentarlos en espacios públicos concurridos.

Una grata sorpresa, consecuencia de los proyectos inclusivos para un conocimiento y cultura de la comunidad sorda fue la iniciativa de algunos profesores de la Facultad de Estadística e Informática quienes se dieron a la tarea de crear una aplicación digital (app) para sordos.

Durante este recorrido se ha constatado y hecho presente que la educación nos brinda muchas posibilidades, muchos caminos; debemos como académicos atrevernos a hacer algo por la inclusión del otro y a favor de un mejor espacio habitable; nuestros estudiantes desde cualquier profesión son un gran potencial para contribuir en ello; nuestro compromiso y ética es facilitarles esas posibles vías.

Por último, podemos comentar el interesante y productivo trabajo de colaboración interdisciplinaria que propició este ejercicio. Además de la relación de diálogo que se propició con autoridades del gobierno del Ayuntamiento local y el apoyo brindado. 


\section{Referencias}

Arnáiz, P. (1996). Las escuelas son para todos, Siglo cero, núm. 27:2, 25 p. educaciones.cubaeduca.cu/medias/pdf/1120.pdf, fecha de consulta: 7 de enero de 2016.

[2] Booth, T. \& Ainscow, M. (2000). Guía para la evaluación y mejora de la educación inclusiva -Index for inclusión. Madrid: Consorcio Universitario para la Educación Inclusiva.

[3] Cruz, M. (2008). Gramática de la Lengua de Señas Mexicana, México, D. F.: CEl Colegio de México, Tesis para optar al grado de Doctor en Lingüística.

[4] Freire, P. (2011). La educación como práctica de la libertad, México, D. F.: Siglo XXI

[5] Giménez, Gilberto. (2005). Teoría y análisis de la cultura, México: CONACULTA.

[6] López, C. (2013). El arte como herramienta de invención y mediación social. Madrid: Grupo 5.

[7] López, M. (2011). Barreras que impiden la escuela inclusiva y algunas estrategias para construir una escuela sin exclusiones, Innovación educativa, núm. 21, pp. 37-54.

[8] Klein, J. (2006). Arteterapia, una introducción. Barcelona: Octaedro.

[9] Mobwa M., Philibert, J. y Olivo N. (2011). Inclusión, accesibilidad, diversidad, igualdad. Guía de acción contra la discriminación, Consejo Nacional para Prevenir la Discriminación: México, D. F.

[10] Ortiz, M. \& Lobato, X. (2003). Escuela inclusiva y cultural, algunas evidencias empíricas. Madrid: Bordón.

[11] Plan de acción para el fortalecimiento de la educación inclusiva. (2005). México, D. F.: SEP.

\section{Electrónicas}

ANUIES. Manual para la integración de personas con discapacidad,

https://www.conapred.org.mx/documentos_cedoc/Manual_inte gracion educacion superior_UNUIES.pdf, fecha de consulta: 18 de abril de 2018 .

Conapred. (2011). Guía ICI, http://www.conapred.gob.mx, fecha de consulta: 7 de enero de 2016.

Cruz, M. (2009). La educación del sordo en México, siglos XIX y XX: La Escuela Nacional de Sordomudos, http://www.cultura-sorda.org/la-educacion-del-sordo-enmexico-siglos-xix-y-xx-la-escuela-nacional-de-sordomudos/, fecha de consulta: 28 de agosto de 2018 .

Diario Oficial de la Federación. (2011). Ley General para la Inclusión de las Personas con Discapacidad, 30 de mayo de 2011, https://www.gob.mx/, fecha de consulta: 7 de enero de 2016.

(2015). Ley General de Turismo, 17 de junio de 2009, https://www.gob.mx/, fecha de consulta: 7 de enero de 2016.

Encrevé, F. (2008). "Réflexions sur le congreso de Milan et ses conséquences sur la langue des signes française à la fin du XIX siècle", Le Mouvement Social 2, $\mathrm{n}^{\mathrm{o}} 223$, https://www.cairn.info/revue-le-mouvement-social-2008-2page-83.htm, fecha de consulta: 18 de abril de 2018.
Giménez, G. La cultura como identidad y la identidad como cultura,

perio.unlp.edu.ar/teorias2/textos/articulos/Gimenez.pdf, fecha de consulta: 8 de enero de 2016 .

INEGI. http://cuentame.inegi.org.mx, fecha de consulta: 7 de enero de 2016.

Jordan, K. Para más señas, Laura, www.conapred,org.mx/kipatlas/K0010.pdf, fecha de consulta: 16 de julio de 2016.

Lasso, M. Cultura inclusiva en la escuela, www.usfq.edu.ec/publicaciones/para el aula/, fecha de consulta: 16 de julio de 2016.

Ley para la integración de las personas con discapacidad del Estado de Veracruz de Ignacio de la Llave. (2010). http://www.ordenjuridico.gob.mx/

Documentos/Estatal/Veracruz/wo45766.pdf, fecha de consulta: 8 de enero de 2016.

Loi $\mathrm{n}^{\circ}$ 2005-102. (2005). Pour l'egalité des droits et des chances, la participation et la citoyenneté des personnes handicapées, https://www.legifrance.gouv.fr, fecha de consulta: 18 de abril de 2018 .

Ministerio de Salud Pública. (1989). Ley $\mathrm{N}^{\circ} 16095$, http://www.impo.com.uy/bases/leyes-originales/16095-1989/1, Fecha de consulta: 18 de abril de 2018.

National Institute on Deafness (NIH) (2017). American Sign Language, https://www.nided.nih.gov/health/american-signlanguage, fecha de consulta: 18 de abril de 2018.

OCDE. (2017). Estudio de la política turística de México, Secretaría de Turismo de México, https://www1.oecd.org/industry/tourism/MEXICO\%20TOURI SM

\%20POLICY\%20REVIEW EXEC\%20SUMM\%20ASSESSM ENT\%20AND\%20RECOMMENDATIONS ESP.pdf, fecha de consulta: 18 de abril de 2018 .

[28] OMS. (2018). Sordera y pérdida de la audición, http://www.who.int/es/news-room/fact-sheets/detail/deafnessand-hearing-loss, fecha de consulta; 18 de abril de 2018.

[29] OMS, Banco Mundial. (2011). Resumen. Informe mundial sobre la discapacidad, http://www.who.int/disabilities/world report/2011/summary e s.pdf?ua=1, fecha de consulta: 18 de abril de 2018 .

[30] OMT. (2016). Turismo accesible para todos. Una oportunidad a nuestro alcance, http://www2.unwto.org/es/content/turismoaccesible, fecha de consulta: 18 de abril de 2018 .

[31] Programa Regional de Educación Inclusiva. (s/f). Documento pdf.

Red de escuelas interculturales. www.escuelasinterculturales.eu, fecha de consulta: 7 de enero de 2016.

[33] UNESCO. (s/f). Declaración Universal de los Derechos Humanos, www.unesco.org, fecha de consulta: 20 de enero de 2016.

[34] Universidad Veracruzana. (2010). Plan Maestro para la Sustentabilidad de la Universidad Veracruzana, https://www.uv.mx/cosustenta/files/2012/09/

PlanMaestroSustentabilidad.pdf fecha de consulta: 12 de noviembre de 2016 . 